\title{
Detection of bacterial pathogens from clinical specimens using conventional microbial culture and 165 metagenomics: a comparative study
}

\author{
Lalanika M. Abayasekara ${ }^{3 *}$, Jennifer Perera ${ }^{1,3}$, Vishvanath Chandrasekharan ${ }^{2,3}$, Vaz S. Gnanam³, \\ Nisala A. Udunuwara ${ }^{3}$, Dileepa S. Liyanage ${ }^{3}$, Nuwani E. Bulathsinhala ${ }^{3}$, Subhashanie Adikary ${ }^{3}$, \\ Janith V. S. Aluthmuhandiram ${ }^{3}$, Chrishanthi S. Thanaseelan ${ }^{3}$, D. Portia Tharmakulasingam ${ }^{3}$, \\ Tharaga Karunakaran ${ }^{3}$ and Janahan $l$ lango ${ }^{3}$
}

\begin{abstract}
Background: Infectious disease is the leading cause of death worldwide, and diagnosis of polymicrobial and fungal infections is increasingly challenging in the clinical setting. Conventionally, molecular detection is still the best method of species identification in clinical samples. However, the limitations of Sanger sequencing make diagnosis of polymicrobial infections one of the biggest hurdles in treatment. The development of massively parallel sequencing or next generation sequencing (NGS) has revolutionized the field of metagenomics, with wide application of the technology in identification of microbial communities in environmental sources, human gut and others. However, to date there has been no commercial application of this technology in infectious disease diagnostic settings.

Methods: Credence Genomics Rapid Infection Detection ${ }^{\mathrm{TM}}$ test, is a molecular based diagnostic test that uses next generation sequencing of bacterial 165 rRNA gene and fungal ITS1 gene region to provide accurate identification of species within a clinical sample. Here we present a study comparing 165 and ITS1 metagenomic identification against conventional culture for clinical samples. Using culture results as gold standard, a comparison was conducted using patient specimens from a clinical microbiology lab.
\end{abstract}

Results: Metagenomics based results show a 91.8\% concordance rate for culture positive specimens and 52.8\% concordance rate with culture negative samples. $10.3 \%$ of specimens were also positive for fungal species which was not investigated by culture. Specificity and sensitivity for metagenomics analysis is 91.8 and $52.7 \%$ respectively.

Conclusion: 165 based metagenomic identification of bacterial species within a clinical specimen is on par with conventional culture based techniques and when coupled with clinical information can lead to an accurate diagnostic tool for infectious disease diagnosis.

\footnotetext{
*Correspondence: manahari@credencegenomics.com

${ }^{3}$ Credence Genomics Pvt. Ltd, 12 - 3/2, Sunethradevi Road, Kohuwala,

Nugegoda, Sri Lanka

Full list of author information is available at the end of the article
} 


\section{Introduction}

In nature and in human disease, bacterial microorganisms are found in complex communities. The need for further understanding the role of different microorganisms on human health led to the inception of the Human Microbiome Project (HMP) [1], which uses metagenomics (i.e. the genetic material within a given sample) to characterize the composition of the microbial community in the human body.

Bacterial infection is among the top ten most common causes of death worldwide [2]. Microbial flora in clinical specimens obtained from different parts of the human body includes a variety of different organisms both pathogenic and non-pathogenic. Traditionally, diagnosis of bacterial or fungal infections relied solely on culture based techniques and culture has been considered the gold standard of pathogen detection. However, some organisms may not be easily detectable by conventional culture methods used in most laboratories due to many factors. In a conventional clinical microbiology laboratory setting, microbial culture of most specimens will be carried out under aerobic conditions. Clinical specimens are not routinely investigated for a variety of pathogens e.g. fungi, anaerobes or rickettsial pathogens unless specifically requested or indicated by the clinical history. Standard culture techniques rely largely on morphological and biochemical characterisation for identification, which can lead to decreased specificity. Also, only a fraction of organisms can be successfully cultured in a multipathogen sample due mostly to various factors such as fastidious growth requirements, non-viable organisms or inhibition of pathogenic organisms due to bacteriocin production by other microbes present in clinical specimens $[3,4]$. These factors make accurate diagnosis and treatment of infections a challenge.

\section{S rRNA and ITS1}

In 1985, Pace et al. published a new revolutionary method of bacterial characterisation [5]. The $16 S$ rRNA gene is a universal gene found in all bacterial chromosomes. In identifying the presence of conserved and variable regions in the $16 S$ rRNA gene for use in phylogenetic identification, this technique has opened up an entirely new horizon for bacterial identification. Since the first introduction of this technique, $16 S$ rRNA based characterisation of bacterial species has been universally accepted as an accurate method of bacterial identification, far superior to morphological or biochemical identification $[6,7]$. In much the same way, ITS1 (internal transcribed spacer 1) of the $18 \mathrm{~S}$ rRNA gene has emerged as a useful genomic marker for identification of fungal species [8]. Similar to $16 \mathrm{~S}$ rRNA, the $18 S$ rRNA gene is ubiquitous among fungal species and contains a mixture of highly conserved regions interspersed with variable genetic regions that facilitates metagenomics identification of fungal species. However, despite the accuracy of $16 \mathrm{~S}$ rRNA and ITS1 based detection, clinical application has been severely limited due to the limitations of Sanger sequencing. Sanger sequencing is the "classical" DNA sequencing technique and uses chain termination method to identify the sequence of bases within a DNA molecule. However, the application of Sanger sequencing is limited to an amplified product of a single DNA molecule. Thus Sanger sequencing limits the identification of pathogens in polymicrobial specimens encountered in clinical settings which will contain DNA molecules from different bacterial species. As a result, application of Sanger sequencing requires clinical isolates being cultured in vitro, extending the limitations of culture based identification to this technique [5].

\section{Next generation sequencing based species identification using 16S rRNA}

Next generation sequencing (NGS) takes DNA sequencing technology to the next level. By parallel sequencing processes, NGS allows the simultaneous sequencing of different DNA fragments while delivering accurate identification results. The combination of a universal gene based species identification and NGS gave rise to a new field known as "metagenomics", where microbial diversity within a sample is defined using the genetic material present [9]. Therefore NGS has contributed to the studying of clinical specimens with a multitude of organisms such as the gut flora and has proven to be a useful tool for microbiome analysis [10]. However, use of metagenomics in clinical microbiology settings with respect to clinical utility has not been comprehensively studied $[5,8]$.

In this study, results of bacterial culture and metagenomic $16 S$ rRNA gene testing were compared to determine the specificity and sensitivity of metagenomics relative to aerobic bacterial culture in a clinical setting.

\section{Credence rapid infection detection ${ }^{\mathrm{TM}}$ (credence RID ${ }^{\mathrm{TM}}$ )}

Credence Rapid Infection Detection $^{\mathrm{TM}}$ is a two-part diagnostic test, that uses partial $16 S$ rRNA and ITS1 gene region of bacteria and fungi (respectively) to identify the microbial composition in a clinical sample, combining molecular amplification with metagenomics identification of species. The V1-V2 region of the $16 \mathrm{~S}$ rRNA gene has enough variability to provide species based identification for use in clinical diagnosis in previous research publications and similarly, the ITS1 gene region in fungi, due to its variability among species, is used for clinical diagnosis $[11,12]$.The amplified gene regions are sequenced using the Ion Torrent Personal Genome Machine (PGM) using the Ion $\mathrm{PGM}^{\mathrm{Tm}} \mathrm{HiQ}^{\mathrm{TM}} \mathrm{OT} 2$ and Ion $\mathrm{PGM}^{\mathrm{Tm}} \mathrm{HiQ}^{\mathrm{Tm}}$ Sequencing kits. Credence Rapid Infection Detection ${ }^{\mathrm{TM}}$ uses semiconductor based NGS for rapid detection of bacterial or fungal detection in clinical specimens. 
The test is carried out in two phases: First, preliminary testing for the presence or absence of bacterial or fungal DNA is carried using fusion primers targeting the V1-V2 region of the bacterial $16 S$ rRNA gene and fungal ITS1 gene region. Custom barcoded primer pools were used for amplification of $16 S \mathrm{rRNA}$ and ITS1 gene regions (Primer sequences are available in Additional file 1: Table S1). All primers included universal primer sequences fused with a key sequence, barcode and adaptor sequences as required for analysis on the Ion Torrent platform. The presence of bacteria or fungi (or both) was recorded within $24 \mathrm{~h}$ of receipt of specimen. Once the presence of bacteria/fungi is confirmed, the amplification products are purified and sequenced. The sequencing data is analysed using proprietary bioinformatics pipelines to identify the composition of organisms within the sample. For the purposes of this study, results obtained using this test method shall hereafter be referred as "metagenomics analysis/results/workflow".

\section{Material and methods Ethics statement}

Application for ethical review was submitted to SIDCER accredited Ethical Review Committee, Faculty of Medicine, University of Colombo (Reference EC-16-134). Study protocol was approved on 18th August 2016.

\section{Specimen collection}

Clinical specimens received from the Microbiology department of Nawaloka Metropolis Laboratory, Nawaloka Hospital, Colombo, an ISO 15189 accredited laboratory was used for the comparative study. The laboratory used standard aerobic culture methods for processing clinical specimens and they conformed to standard protocols published for clinical microbiology laboratories for detection of pathogens and interpretation of results [13, 14]. Samples from non-sterile sites were incubated overnight. Sterile fluids were cultured on agar plates (blood, chocolate and Maconkeys agar) and BHI broth. Plates were incubated for $48 \mathrm{~h}$; if no growth was observed at $24 \mathrm{~h} \mathrm{BHI}$ broth was sub-cultured for upto 5 days. Blood cultures were incubated for 5 days routinely or upto 14 days where enteric fever or Brucellosis is suspected (or as per request from physician). Species identification for Gram negative bacilli were carried out using RapID $^{\text {rm }}$ system (remel, Thermo Fisher Scientific).

The remaining or left over specimens (that had been processed for bacterial culture) were stripped of patient identification details and coded before being included into the study. A total of 103 specimens were transferred in batches of 10-12 in ice to the laboratory for metagenomic $16 S$ /ITS1 analysis. The samples were selected from the sample entry register using a random number table over ten consecutive days. The specimens tested are listed in Additional file 2: Table S2. If there was no remaining sample in the selected specimen, the number was skipped and next number in the table was used for selecting the specimen. The researchers in the NGS laboratory were blinded to the microbial culture results. Metagenomic 16S/ITS1 identification was carried out using the Credence Genomics Rapid Infection Detection ${ }^{\text {TM }}$ test.

The following steps were used in the workflow leading to species identification using NGS.

\section{DNA extraction}

As the first step DNA extraction of fungal and bacterial DNA from each specimen was carried out using the QIAAmp DNA Mini kit (Qiagen) according to manufacturer's instructions. Each batch of specimens were extracted with negative buffer control (extraction control).

\section{Library preparation}

The presence or absence of DNA was confirmed through PCR amplification of the bacterial $16 S$ gene V1-V2 region and fungal ITS1 gene region. PCR reactions were prepared in a laminar flow PCR work station with all material UV irradiated prior to use. $12.5 \mathrm{ul}$ of Platinum PCR supermix (Invitrogen) and 2.5ul of each primer pool was added to a final reaction volume of $25 \mathrm{ul}$. PCR was conducted using $12.5 \mu \mathrm{M}$ of each primer and $3.75 \mu \mathrm{l}$ of template DNA.

PCR Amplification was carried out using the following cycle conditions:

$95{ }^{\circ} \mathrm{C}$ for $5 \mathrm{~min}, 10$ cycles at $95{ }^{\circ} \mathrm{C}$ for 30 s, $58{ }^{\circ} \mathrm{C}$ for 30 s and $72{ }^{\circ} \mathrm{C}$ for 60 s, followed by 35 cycles for $95{ }^{\circ} \mathrm{C}$ for $30 \mathrm{~s}, 68^{\circ} \mathrm{C}$ for $30 \mathrm{~s}$ and $72{ }^{\circ} \mathrm{C}$ for $60 \mathrm{~s}$ and one cycle at $72{ }^{\circ} \mathrm{C}$ for $10 \mathrm{~min}$ for $16 \mathrm{~S}$ amplification; and $95{ }^{\circ} \mathrm{C}$ for 5 min, 10 cycles at $95{ }^{\circ} \mathrm{C}$ for $30 \mathrm{~s}, 55^{\circ} \mathrm{C}$ for 30 s and $72^{\circ}$ $\mathrm{C}$ for $60 \mathrm{~s}$, followed by 35 cycles for $95{ }^{\circ} \mathrm{C}$ for $30 \mathrm{~s}, 68{ }^{\circ} \mathrm{C}$ for 30 s and $72{ }^{\circ} \mathrm{C}$ for 60 s and one cycle at $72{ }^{\circ} \mathrm{C}$ for $10 \mathrm{~min}$ for ITS1 amplification respectively. Specimens were run in batches of 10 with positive and negative buffer controls for bacterial and fungi respectively. PCR products were run on a $2 \%$ agarose gel and visualized using ethidium bromide. Specimens were run in batches of 10 with 50 bp ladder, extraction (negative buffer controls), positive controls and PCR blank. E coli ATCC No.25922 and. C. albicans ATCC No 10231 strains were used as positive controls.

Where PCR inhibition was observed this was confirmed by conducting a PCR reaction with the specimen and $1 \mathrm{ul}$ of positive control added. No amplification even in the presence of positive control was taken as confirmation of PCR inhibition.

Final library products were purified using 0.9X of Agencourt AMPure (Beckman Coulter) beads according to manufacturer's instructions, eluted in low $\mathrm{TE}$ and quantified using the Qubit dsDNA HS Assay kit (Invitrogen). 
Specimen were pooled in batches of 20 and sequenced using the Ion Torrent $\mathrm{PGM}^{\mathrm{sx}}$ platform. The sequence data was analysed using a proprietary bioinformatics pipeline that maps the reads sequenced to a phylogenetic tree with the entire microbial profile within the sample tested.

\section{Semi-conductor sequencing}

Template preparation and sequencing of final libraries was conducted on the Ion OneTouch 2 system and Ion PGM using Ion PGM ${ }^{\mathrm{m}} \mathrm{Hi}-\mathrm{Q}^{\mathrm{m}}$ OT2 Kit (Thermo Fisher Scientific) and Ion PGM ${ }^{m}$ Hi-Q ${ }^{m}$ Sequencing Kit (Thermo Fisher Scientific) on the Ion Torrent Personal Genome Machine (PGM) according to manufacturer's instructions. Barcoded bacterial and fungal libraries were multiplexed on a single chip on a 400 bp run to obtain sequencing data. Specimens were run in batches of 20 on an Ion $318^{\text {mo }}$ v2 chip (Thermo Fisher Scientific).

\section{Data processing and bioinformatics}

Data was analysed using Credence Genomics proprietary bioinformatics pipeline for analysis of clinical isolates. Reads obtained from sequencing run were trimmed, removing barcode and adaptor sequences. After trimming, quality control parameters (Phred Quality Score cut off and minimum read length) for all sequence data were checked. A minimum of 2000 reads per specimen were selected, with cut offs at Phred quality score 16; fragment length > $300 \mathrm{bp}$ for bacterial reads and $>200$ bp for fungal reads. FASTQ formats generated were mapped to the NCBI-RefSeq (26:09:2016) database using Credence Infectious Panel Pipeline 1.1.0 (Credence Genomics). FASTA files and phylogenies generated from the bioinformatics pipelines are available in Additional files 3 and 4 respectively.

\section{Phylogenetic output \& relative abundance}

Results of metagenomic analyses were compiled according to results from phylogenetic mapping. Relative abundance for each organism was calculated based on number of reads mapped for each species as a percentage of the root read value, with the species with the largest percentage of reads classified as the species of highest abundance. However, relative abundance data was not used for the final analysis of the specimens. Figure 1 shows the phylogenetic trees from 3 different samples.

\section{Analysis of culture negative specimens}

When culture negative results were positive for 16S PCR results, the libraries generated were subjected to metagenomic analysis to determine the species of the bacteria detected.

\section{Results}

Total number of specimens and specimen types analysed in this study are shown in Fig. 2. For the purpose of this validation, bacterial culture results where available were considered as the base line for comparison. Culture negative specimens where PCR based detection was positive were reviewed by a medical microbiologist for possible correlation of clinical data with metagenomic analysis. Final analysis of results was possible for a total of 97 specimens out of the 103 samples compared (Fig. 2).

\section{Assessment criteria}

Specimens were considered positive for bacteria/fungi if expected bands were observed in the PCR reaction ( $>300$ bp and $>200$ bp respectively). Library contamination was identified by the presence of bands in the extraction controls or PCR blank. Contaminated specimen(s) were excluded from the final analysis. Positive samples were sequenced and bioinformatics analysis carried out. Specimens which did not pass the quality parameters for bioinformatics analysis were considered conflicts in the final analysis.

For the purposes of the validation, culture results of specimens were provided the default status as accurate and the results of the metagenomic analysis were compared against the culture results. As a result, it was expected that at least culture negative results would show discrepancies when compared against the metagenomic results (due purely to the likely presence of anaerobic bacteria, slow growing organisms, fastidious organisms etc. which would not have been detected during conventional bacterial culture). However, these too were considered conflicts in the final analysis of results.

Where species/genera of culture isolates were detected in the metagenomic analysis, this result was designated as a "match". The results of comparisons were designated as "conflicts" when species (or species classification as described in the culture report i.e. "coliform organisms", "Staphylococcus spp.", etc.) isolated in culture were not detected in metagenomic testing or when culture negative specimens were positive for bacterial species in the metagenomic workflow. The species identified in the metagenomic workflow were assessed for clinical significance by a microbiologist in order to verify the significance of the positive metagenomic results based on clinical history.

From the 103 specimens received for analysis, 5 blood culture specimens (P8, P9, P18, P19 and P52) showed signs of inhibition after the first DNA extraction and PCR reactions were resolved on the agarose gel (i.e. absence of amplified bands and primer dimers). Other specimen processed in the same batch with the blood culture specimens showed no sign of inhibition. After PCR inhibition was confirmed (as described in Methods), based on a literature review on PCR inhibitors in blood culture, it was concluded that inhibition was possibly due to components in the blood culture medium [15]. 


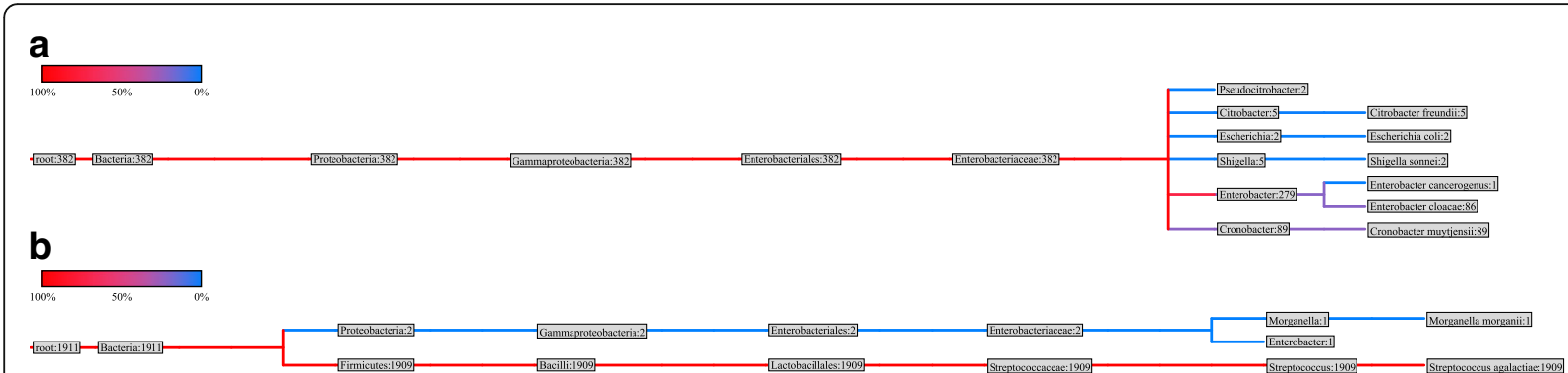

C

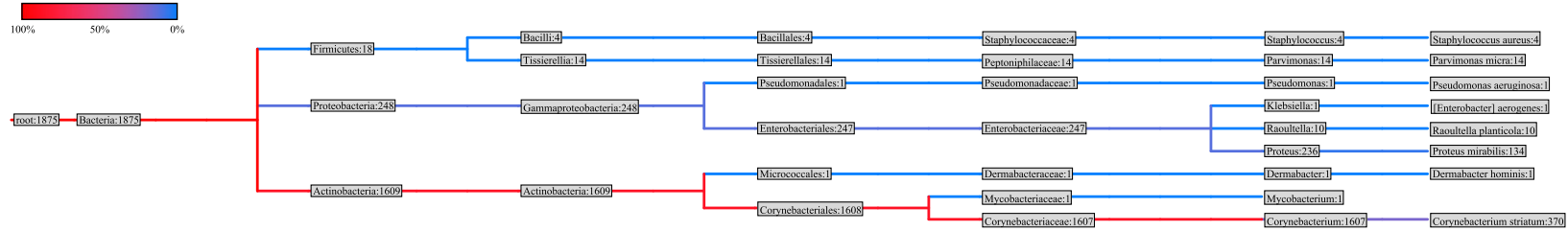

Fig. 1 Phylogenetic tree of urine specimen (a) nasogastric aspiration specimen (b) and pus swab (c). Numbers displayed next to the species in the final branches of the phylogenetic tree indicate the number of reads successfully aligned to the reference $16 \mathrm{~S}$ rRNA sequence of this species

One specimen (P56) could not be analysed in the metagenomic workflow due to contamination and was excluded from the analysis process, resulting in a final comparative analysis of 97 specimens.

\section{Overview of results comparison}

Of the 97 specimens processed using metagenomic analysis, 36 specimens were reported as no bacterial growth (NBG) and were culture negative; 61 specimens were culture positive. A comprehensive list with individual specimen results, culture to NGS comparison, is available in Additional file 2: Table S2. Fig. 1 shows an example of the final format of the phylogenetic tree mapped after bioinformatics analysis.

Comparison of $\mathrm{PCR} /$ metagenomics results with bacterial culture outcomes are shown in Table 1 . Of the 61 culture positive specimens, 60 were positive at PCR level (Table 1) and 56 of these metagenomics results matched with culture

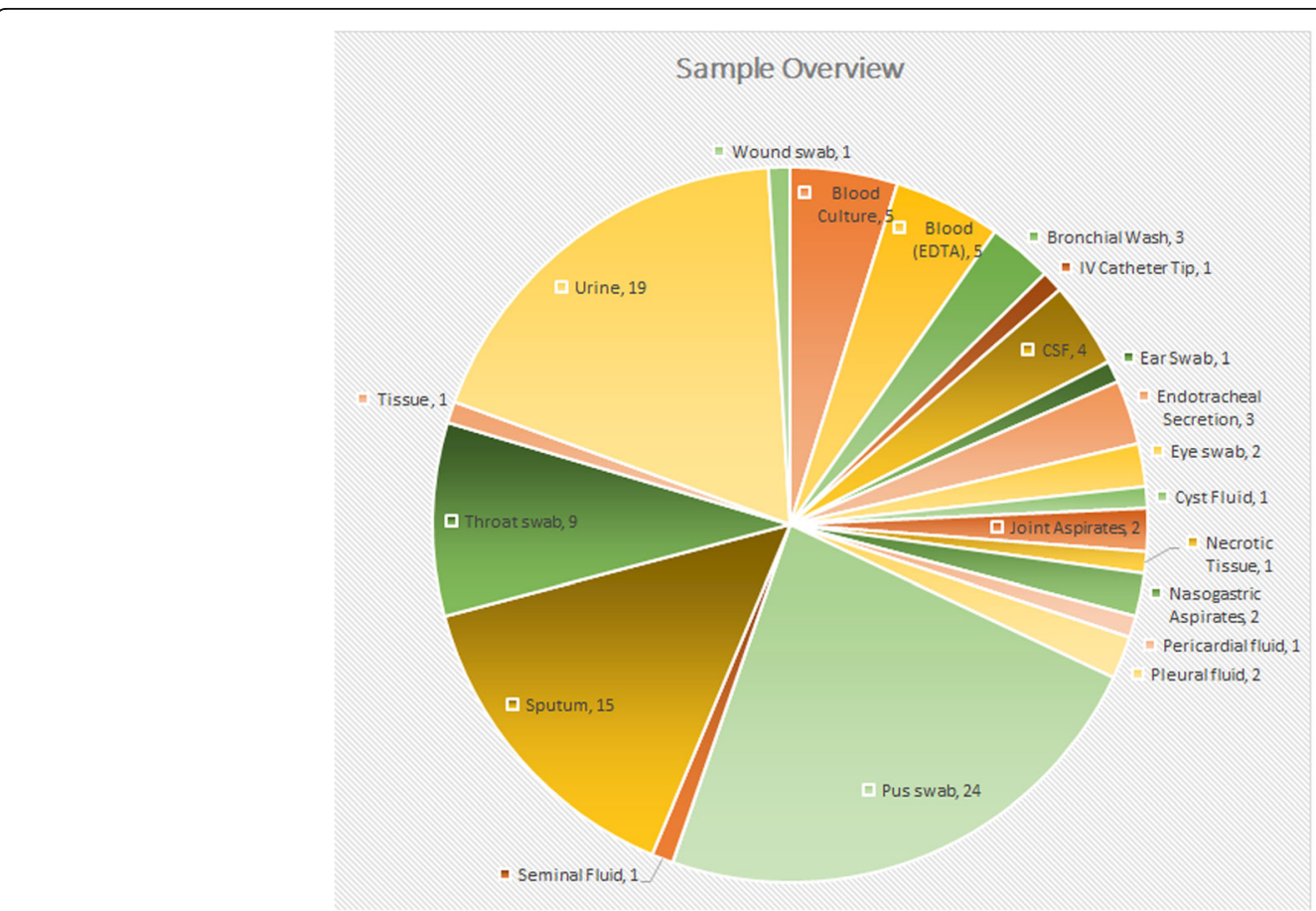

Fig. 2 The types and numbers of different clinical specimens used in the analysis $(n=103)$ 
Table 1 Comparison of culture results vs PCR results

\begin{tabular}{|c|c|c|c|c|}
\hline Total Specimens & 103 & & & \\
\hline \multirow[t]{3}{*}{ NGS processed specimens } & 97 & & & \\
\hline & & \multirow[t]{2}{*}{ Total Specimens } & \multicolumn{2}{|c|}{ Bacterial PCR Results } \\
\hline & & & Negative & Positive \\
\hline \multirow[t]{2}{*}{ Culture results } & No Bacterial Growth & 36 & 19 & 17 \\
\hline & Culture Positive & 61 & 1 & 60 \\
\hline
\end{tabular}

results either at species level $(n=3)$ or genus level $(n=53)$. Of the culture negative specimens processed using the metagenomic workflow, $52.8 \%(n=19 / 36)$ matched with culture results (Table 2).

\section{Analysis of culture positive results}

A total of 61 specimens were culture positive and of these 56 had species that were identified by the metagenomics workflow, giving a match rate of 56/61 (91.8\%) (see Table 2). Details of specimens with conflicting results are shown in Table 3. For conciseness, where metagenomic workflow identified a large number of detected species, only species of the highest abundance and/or clinical relevance are displayed.

\section{Analysis of culture negative results}

A total of 36 culture negative specimens were received and analysed by metagenomic workflow. Of these, 19 specimens were also negative from the metagenomic workflow, which left 17 conflicting specimens where PCR based detection was positive but culture negative (hereafter referred to as 'PPCN' specimens) (Table 4). As with Table 3, where a large number of species were identified in the metagenomic workflow only species of highest abundance and/or clinical relevance is displayed.

\section{Fungal results}

As standard practice, Credence Genomics metagenomic workflow also uses ITS1 region of fungal DNA for detection and identification of fungal species. 10 specimens were positive for fungal identification by metagenomics analysis. The species isolated from fungal metagenomics are listed in Table 5 (only species of highest abundance displayed). However, these specimens were not tested by fungal culture in a routine Microbiology laboratory.

\section{Discussion}

Next Generation Sequencing (NGS) based identification of bacterial and fungal species has been widely available for the last decade and increasingly attention has been focused on clinical applications of this technique [16, 10]. The versatility of NGS technology allows application of $16 S$ identification to uncultured clinical samples, resulting in fast, comprehensive analysis of the microbial profile within a clinical sample. One of the greatest advantages of this technology is the universal coverage of all medically relevant bacteria and fungi in a single test. In this study we describe a commercial diagnostic product offered by Credence Genomics Pvt. Ltd. as part of routine clinical diagnostics for universal detection and identification of fungal and bacterial clinical species and validation of the said product against conventional bacterial culture.

In order to establish a baseline accuracy for validation of this product, bacterial culture specimens were compared against the results of the partial $16 S$ based metagenomic identification.

\section{Results concordance for bacterial metagenomic results}

$60 / 61$ culture positive specimens were also positive for bacteria in the metagenomics analysis library preparation (PCR). 56/60 specimens were matches when sequenced and analysed; giving a total concordance of 56/61 for culture positive specimens.

The conflicting results in P25, P29, P70, P75 and P97 (see Table 3) cannot be resolved without further validation or querying the microbiological identification process. While the absence of metagenomic identified species among culture isolates may be attributed to decreased sensitivity, slow growth, growth inhibition, microbial interaction or even the growth conditions; the failure of the metagenomics workflow to identify culture species/isolates cannot be explained since conventional wisdom indicates that PCR based testing would be more sensitive than culture. However, since identification of species in conventional culture requires visual

Table 2 Comparison of culture results and bacterial metagenomic results

\begin{tabular}{llll}
\hline & Culture Negatives (\%) & Culture Positives (\%) & Total Specimens \\
\hline Matches with metagenomic results & $19(52.8 \%)$ & $56(91.9 \%)$ & $75(77.3 \%)$ \\
Conflicts with metagenomic results & $17(47.2 \%)$ & $5(8.1 \%)$ & $22(22.7 \%)$ \\
Total & $36(100 \%)$ & $61(100 \%)$ & $97(100 \%)$ \\
\hline
\end{tabular}


Table 3 The details of culture positive specimens with conflicting results

\begin{tabular}{|c|c|c|}
\hline $\begin{array}{l}\text { Specimen } \\
\text { Type }\end{array}$ & Culture Results & $\begin{array}{l}\text { Metagenomic Results (no. of } \\
\text { species within the genus) }\end{array}$ \\
\hline $\begin{array}{l}\text { Sputum } \\
(P 25)^{c ~ a ~}\end{array}$ & Coliforms & $\begin{array}{l}\text { Porphyromonas pasteri }{ }^{\mathrm{b}} \\
\text { Streptococcus species. (9) } \\
\text { Prevotella species (10) }\end{array}$ \\
\hline $\begin{array}{l}\text { Throat } \\
\text { Swab } \\
(\text { P29) }\end{array}$ & Moraxella spp & $\begin{array}{l}\text { Prevotella species (9) } \\
\text { Prevotella melaninogenica }{ }^{\text {b }}\end{array}$ \\
\hline $\begin{array}{l}\text { Pus Swab } \\
\text { (P70) }\end{array}$ & $\begin{array}{l}\text { Coagulase negative } \\
\text { Staphylococcus species }\end{array}$ & $\begin{array}{l}\text { Streptococcus } \\
\text { Filifactor alocis } \\
\text { Prevotella intermedia }\end{array}$ \\
\hline $\begin{array}{l}\text { Pus Swab } \\
\text { (P75) }\end{array}$ & Acinetobacter spp & $\begin{array}{l}\text { Prevotella bivia } \\
\text { Peptoniphilus indolicus } \\
\text { Finegoldia magna } \\
\text { Dialister micraerophilus } \\
\text { Veillonella montpellierensis } \\
\text { Streptococcus anginosus } \\
\text { Ureaplasma parvum }\end{array}$ \\
\hline $\begin{array}{l}\text { Pus Swab } \\
\text { (P97) }\end{array}$ & Coliform Organisms & $(-)$ \\
\hline
\end{tabular}

${ }^{a}$ Samples in which all species detected in metagenomics workflow are not displayed due to the large number of species identified. See Additional file 2 for all species identified per sample

${ }^{b}$ species of highest abundance

'fungal species detected

identification, we can speculate that human error could account for misidentification of morphologically similar organisms leading to the conflicts seen here.

The conflict seen with P97 is also difficult to explain as the metagenomics results of the specimen shows negative whereas culture has isolated coliforms from this sample. While it is possible to argue that the culture maybe showing a false positive due to contamination, it is equally likely that the bacterial load present in the sample may be below the analytical sensitivity threshold of the metagenomic test, which results in a false negative from the metagenomics analysis.

The minor conflict is seen with the metagenomics results culture results in specimen P58 is due to the difference in the coagulase activity of the species. Other instances where culture isolates were reported as coagulase negative Staphylococcus species were correctly reflected in metagenomic results. Out of a total of 8 Staphylococcus isolates identified by culture, 6 correlated correctly with metagenomics detected species. Given the sensitivity of metagenomic identification, it is extremely likely that this conflict in reporting of coagulase activity is due to the limitations of the tube test used to detect coagulase activity, as absence of appropriate plasma controls or shortened reaction time may have led to culture based misidentification [17]. However, as per the assessment criteria, the culture isolates match at genus level with the metagenomic analysis and is therefore designated as matches.

Out of 17 PPCN (PCR positive, culture negative) specimens (see Table 4), 2 (P1, P5) failed to meet the quality
Table 4 Metagenomic results of PCR Positive, Culture Negative (PPCN) specimens

\begin{tabular}{lll}
\hline $\mathrm{SRN}$ & Specimen Type & NGS results \\
\hline $\mathrm{P}^{\mathrm{c}}$ & Urine & $\begin{array}{l}\text { Enterococcus faecalis*, Enterococcus } \\
\text { phoeniculicola }\end{array}$ \\
$\mathrm{P} 5^{\mathrm{c}} \quad$ Urine & $\begin{array}{l}\text { Methylobacterium longum } \\
\text { sphaerophysae, Burkholderiale, Paracoccus } \\
\text { sphaerophysae, Aquabacterium parvum, Bacillus } \\
\text { amyloliquefaciens, Kytococcus aerolatus }\end{array}$
\end{tabular}

\begin{tabular}{|c|c|c|}
\hline$P 14^{d}$ & Urine & $\begin{array}{l}\text { Morganella morganii*, Stenotrophomonas } \\
\text { maltophilia }\end{array}$ \\
\hline$P 23^{d}$ & CSF & Ralstonia ${ }^{\mathrm{a}}$ \\
\hline$P 24^{d}$ & Pleural Fluid & Tessaracoccus * \\
\hline P30 $0^{d}$ & Pus Swab & $\begin{array}{l}\text { Finegoldia magna*, Pseudomonas aeruginos } \\
\text { (low abudance), Staphylococcus aureus (low } \\
\text { abundance) }\end{array}$ \\
\hline$P 34^{d}$ & Urine & Prevotella bivia*, Streptococcus infantis \\
\hline $\begin{array}{l}P 37^{b}, \\
d\end{array}$ & $\begin{array}{l}\text { Endo tracheal } \\
\text { secretion }\end{array}$ & $\begin{array}{l}\text { Streptococcus a, Streptococcus parasanguinis, } \\
\text { Streptococcus mitis, Raoultella planticola, } \\
\text { Porphyromonas gingivalis }\end{array}$ \\
\hline
\end{tabular}

P40 ${ }^{d}$ Pus Swab Rhizobiales ${ }^{\mathrm{a}}$, Corynebacterium, Prevotella bivia, Corynebacterium tuberculostearicum

$\mathrm{P} 1^{\mathrm{d}}$ Pus Swab Corynebacterium*, Jonquetella anthropi, Staphylococcus (low abundance)

P53 Cyst fluid Micrococcus luteus ${ }^{\mathrm{a}}$, Streptococcus spp., Staphylococcus spp.

P54 CSF Mycoplasma hominis, Comamonas denitrificans

P57 $^{\text {d Wound Swab Phyllobacteriaceae }}{ }^{\text {a }}$, Sphingopyxis fribergensis, Staphylococcus spp. (9), Corynebacterium (6)

P59 ${ }^{d}$ Pleural Fluid Staphylococcus aureus ${ }^{\mathrm{a}}$, Sphingopyxis fribergensis

$\mathrm{P} 60^{\mathrm{d}}$ Sputum Comamonas denitrificans ${ }^{\mathrm{a}}$, Acinetobacter baumannii, Streptococcus spp. (5), Staphylococcus spp. (5)

P67 ${ }^{d}$ Pus Swab Staphylococcus aureus ${ }^{\mathrm{a}}$, Burkholderia multivorans

$\mathrm{P} 81^{\mathrm{b}}$, Seminal Fluid Prevotella bivia*, Staphylococcus intermedius, d Staphylococcus petrasii, Lactobacillus fermentum

Anaerobic species are highlighted in bold text ${ }^{*}$

a species of highest abundance

bfungal species detected

'Sequencing output did not pass the QC parameters for

bioinformatics analysis

${ }^{\mathrm{d}}$ Samples in which all species identified in the metagenomic analysis are not displayed due to the large number of species identified. See Additional file 2 for all species identified per sample

parameters for successful bioinformatics analysis (i.e. less than 2000 reads of $>300$ bp at Phred quality score 16) and were designated as conflicts. Though these specimens showed successful amplification of expected bacterial fragment at the library preparation step, the concentration of the purified library was too low for successful sequencing and subsequent bioinformatics analysis. Metagenomic results for 8 specimens (P14, P24, P30, P34, P40, P51, P57 and P81) can be readily explained as to why these specimens were reported as culture negative. These specimens predominantly contain anaerobic bacteria which would not grow in standard aerobic culture media as was used in 
Table 5 Total fungal species identified using ITS1 metagenomic workflow

\begin{tabular}{ll}
\hline $\begin{array}{l}\text { Specimen type (number of specimens with } \\
\text { identified species) }\end{array}$ & Fungal NGS Species \\
\hline Throat Swab (1), Sputum (1), Seminal fluid (1), & Candida albicans $^{\mathrm{a}}$ \\
Sputum (1), Bronchial wash (1) & Candida tropicalis $^{\mathrm{a}}$ \\
Throat Swab (1) & $\begin{array}{l}\text { Lomatium }^{\mathrm{n}} \\
\text { nevadense }\end{array}$ \\
Endotracheal secretion (1) & $\begin{array}{l}\text { Saccharomyces } \\
\text { cerevisiae }^{\mathrm{a}}\end{array}$ \\
Pus Swab (1) & Uwebraunia $^{\mathrm{a}}$ \\
Pus Swab (1) & Candida \\
& orthopsilosis $^{\mathrm{a}}$ \\
\hline
\end{tabular}

${ }^{\mathrm{a}}$ species of highest abundance

this case. Additionally, M. hominis detected in another specimen (P54), is a fastidious organism, which would typically take up to 4 days to grow and lacks a cell wall which makes Gram staining and morphological identification difficult $[18,19]$. Therefore, these 9 metagenomic workflow results in context can be attributed as false negatives in culture. Furthermore, fungal metagenomics showed that 2 specimens (P37 and P81) had fungal species (Saccharomyces cerevisiae and Candida albicans respectively). Inhibition of bacterial culture growth due to the presence of fungal species is a distinct possibility, and can be considered as an example of the limitation of bacterial culture. Therefore, out of a total of $17 \mathrm{PPCN}$, the discrepancies of 10 specimens (P14, P24, P30, P34, P37, P40, P51, P54, P57 and P81) can be attributed to the limitations of standard culture methods.

Literature review of $16 \mathrm{~S}$ sensitivity against culture negative specimens, shows on average that $50 \%$ of culture negative specimens will be reported as positive by metagenomics/16S PCR analysis [20-22]. The metagenomic results of culture negative specimens reported in this study, were further analysed for clinical validity by the clinical microbiologist. It was finally concluded, that results of PPCN specimens may be due to increased sensitivity of the metagenomics work flow, which resulted in unviable or low bacterial load being detected. But in the absence of clinical follow up in real time, it is not possible to confirm the NGS findings at this point in time and is considered a limitation of this study.

\section{Final concordance values for bacterial Metagenomics}

Based on the analysis conducted, it was found that metagenomics results have a concordance rate/positive predictive value of $91.8 \%(56 / 61)$ when compared with culture positive specimens. It covers a wider range of aerobic and anaerobic bacteria and provided species level identification where conventional culture could not. However, it should be noted that metagenomics results can give a wide range of organism and microbial profile can be difficult to interpret. Some of the matching specimen have a large number of bacterial species identified and though culture isolates will also be present in the metagenomics results, in some cases it will be present at a very low abundance (i.e. the percentage of read sequenced from this organism relative to all reads sequenced within the sample is low,

Using stringent comparison criteria for all specimens analysed in the study, the concordance rate was 77.3\% $(n=75$ / 97) for metagenomics vs. culture comparison. However, based on the limitation of conventional aerobic culture and already documented error rates of false negatives in culture (i.e. assuming at least $50 \%$ of culture negatives as false negatives attributes another 10 specimens as matches), concordance rate is likely closer to $87.6 \%(n=85 / 97)$. Based on clinical review the conflicts in the PPCN specimens can be attributed to the higher sensitivity of metagenomic-based identification of species commonly detected in respective clinical settings in relation to each particular specimen. When PCR results of PPCN specimens are included in the analysis in addition to culture positive specimens the concordance rate increased to $94.8 \%$.

Though there was no corresponding fungal culture data for validation of fungal results, it is important to note that 10 of 97 specimens (10.3\%) were positive for fungal detection which would routinely be missed in a standard clinical setting. The primary fungal species detected was Candia albicans with other less common Candida species (see Table 5) and clinical sites being mainly throat swabs and endotracheal secretions which may have been ignored during culture interpretation at the Microbiology work bench.

\section{Specificity and sensitivity}

By using culture results as the base line for the presence or absence of disease, the specificity of and sensitivity of the Credence RID ${ }^{\text {tw }}$ test can be calculated, using the assessment criteria as mentioned above, the sensitivity and specificity of the test is $91.8 \%(n=56 / 61)$ and $52.8 \%$ (19/36) respectively.

\section{Conclusions}

\section{Commercial clinical Metagenomics-based bacterial identification}

The profile of bacteria within a clinical specimen can vary widely based on treatment history of the patient and site of sampling (Fig. 1). As evidenced by the specimens in this study, non-sterile clinical sites, such as throat, respiratory tract or skin can often demonstrate more than 20 different bacterial species. Therefore, clinical interpretation of metagenomic based results of bacterial and fungal identification requires careful analysis of symptoms and the clinical relevance of each organism identified. This can make the application of metagenomic as the sole clinical diagnostic tool challenging, especially where clinical details on 
the patient's condition are not available. Therefore, application of metagenomics analysis in the clinical setting will require thorough knowledge on the patient's condition and clinical history. When compared to conventional diagnostic tests which provide a straight yes/no result, metagenomic diagnostics is far more complex. However, if specimen collection and clinical correlation as carefully conducted, metagenomics diagnostics can be universally applied for elimination of a suspected pathogen, for confirmation of a suspected pathogen or for a broad screening of where there is no suspected pathogen.

The commercial metagenomic diagnostic product offered by Credence Rapid Infection Detection ${ }^{\text {Th }}$ has proven to be clinically applicable and has the ability to identify a superior range of organisms. The use of the universal $16 S$ rRNA for bacterial species identification has already been established as more accurate than culture $[6,7]$. Its application has previously been hindered by the limitations of sanger sequencing and inability to design a rapid method of detection, which has now been addressed with the commercial availability of parallel sequencing platforms.

The outcome of this study shows that clinical metagenomics is at very least comparable to bacterial culture. However, the natural limitations in attributing culture as the default for the gold standard of diagnostic detection has resulted in a culture biased outcome for this study. The limitations of the culture technique cannot be ignored in a routine diagnostic setting as most specimens will be processed by aerobic culture methods unless specifically indicated, providing a relatively inaccurate diagnostic test result. This would naturally result in false negative cultures where anaerobic pathogens are present in the specimen as demonstrated by the metagenomic based results of this study. The additional limitations of growth conditions, growth rates of organisms or even bacteriocins produced by polymicrobial growth can easily alter the results of bacterial culture testing.

In comparison, a culture independent test such as $16 S$ based metagenomic identification is not limited by these considerations and can rapidly identify a wide range of organisms. Based on the wide range of organisms identified and the speed and high throughput capacity of metagenomics, it is clear that it can be a powerful clinical tool for diagnosis. The ability of the test to provide information on relative abundance of the varied organisms in the specimens adds further value to the report.

With regard to the clinical application of this test, a detailed output of microbial composition of a specimen provides an opportunity to the infectious diseases specialists to analyse the patient situation in a more comprehensive manner. However, this wider coverage of bacterial species naturally leads to complex reporting profiles which requires close correlation with patient symptoms and clinical judgement for application.

\section{Limitations of the credence rapid infection detection ${ }^{\mathrm{Tm}}$ test}

Out of a total 103 culture specimen received for analysis in the NGS laboratory, 6 specimens were excluded from the final analysis. 5 of these specimens were blood culture specimens. Processing of these specimens showed PCR inhibition, possibly due to the presence of sodium polyanetholesulfonate which is a known inhibitor of PCR. [15]. This has implications for clinical application of this diagnostic test as specimens that are already submitted for blood culture cannot be analysed without significant changes made to the test process. To mitigate this limitation, blood specimens must be collected into sterile EDTA containers instead, for analysis.

Secondly, the chance of incidental contamination of the specimen upon collection is very high, and indeed has been observed by the Credence Genomics on routine clinical testing (anecdotal evidence). Collection of blood, CSF and other sterile fluids can easily be contaminated by bacterial skin flora if the collection process is not carried out aseptically or the specimen is collected from a catheter/cannula site. The presence of contaminating bacteria, especially skin flora while not affecting the sequencing output (contaminating bacteria will simply be displayed along with all other bacterial species in the specimen), can affect clinical application of the test as the abundance values would be skewed due to the presence of the contaminating bacteria. Furthermore, the library preparation process is highly susceptible to contamination by amplicons and rigorous procedure must be used to ensure that all specimens pass the necessary quality controls.

Thirdly, the limitations in the curation of the bioinformatics databases can have an impact on the results outcome. The Credence RID ${ }^{\text {ts }}$ test uses the NCBI Refseq, a rapidly expanding, curated database with $16 S$ sequences curated for 17,654 bacterial species and ITS1 sequences curated for 5365 fungi species [23]. However, database curation is a long and tedious process and new variations in existing species classifications or novel species identification can take a long time. Therefore, the results of the test are limited by the accuracy and scope of the existing Refseq database.

\section{Limitations of this study}

As a comparative study using bacterial culture as the standard for comparison there are a number of limitations to be addressed. Firstly, this study only uses the culture results from a clinical microbiology lab where only aerobic culture is routinely conducted. As explained previously, clinical microbiology labs in Sri Lanka will only routinely 
culture for aerobic bacteria unless specifically requested for a wider range by the physician. As a result, the culture isolates and culture negative specimen can be hypothesized to indicate the presence on anaerobic species or slow growing, fastidious organisms. Furthermore, species based identification in bacterial culture is also limited due to inaccuracy of biochemical testing and growth inhibition/ competition that can occur in polymicrobial specimens. This hampers the interpretation of metagenomic based analysis, particularly in the case of conflicts in culture isolates and culture negative specimen.

Secondly, there is no information on the fungal culturing for these specimens. The presence of fungal organisms as detected by the metagenomic workflow can inhibit the growth of bacterial culture. However, without fungal culture results, there is no independent verification of the fungal results from the metagenomic analysis.

The above limitations, results in a biased measurement of culture vs. metagenomic identification of bacteria. For a truly accurate representation of the sensitivity and accuracy of metagenomic identification, a clinical correlational study with patient follow up and treatment would have to be conducted to ensure that the outcome of the metagenomic analysis is a true representation of bacterial flora within a specimen.

\section{Additional files}

Additional file 1: Primer sequences. Primer sequences used for amplification of the bacterial 165 rRNA V1 - V2 region and fungal ITS1 region respectively (Barcode and adaptor sequences are not included). (DOCX $12 \mathrm{~kb}$ )

Additional file 2: Details of specimen type, culture results and metagenomic results. All specimens analysed are described in this table. Specimens where metagenomic results match culture results; reference number cells highlighted in green. Specimens where metagenomic results conflict with culture results; reference number cells highlighted in red. Specimens that were excluded from the final analysis; reference number cells highlighted in black. Specimen where metagenomic result cell is highlighted in light grey; all species identified in the metagenomic workflow are not listed due to the large number of species identified. Species listed are based on species that match culture results, abundance (i.e. high abundance) and clinical relevance (i.e. low abundance). Specimens where metagenomics results conflict with culture negative results; reference number cells in white. Genus (n); multiple species of this genus identified in the metageomics workflow. The genus and number of species within that genus ( $\mathrm{n}$ ) displayed. ${ }^{*}$ species of highest abundance. Bold species that match culture results. (DOCX $27 \mathrm{~kb}$ )

Additional file 3: QC passed FASTA files generated from the Credence Infectious Panel Pipeline 1.1.0. Unaligned BAM files generated from the sequencing run were trimmed of barcode and adaptor sequences and this quality checked for the following QC parameters: Phred quality score 16; fragment length $>300$ bp for bacterial reads and $>200$ bp for fungal reads respectively and final FASTA sequences obtained. Specimen files are named as follows: Additional file 3_B/F_Specimen number (e.g. Additional file 3_B_P1 - fasta files of bacterial sequences within specimen P1; Additional file 3_F_P1- fasta files of fungal sequences within specimen P1). (PDF $37 \overline{5} 7 \overline{5} \mathrm{~kb}$ )
Additional file 4: Final phylogenies generated from the Credence Infectious Panel Pipeline 1.1.0. Phylogenies generated from the quality checked FASTA files for each specimen is available here. Specimen files are named as follows: Additional file 4_B/F_Specimen number (e.g. Additional file 4 B P1- phylogeny of bacterial sequences within specimen P1; Additional file 4_F_P1 - phylogeny of fungal sequences within specimen P1). (PDF $620 \mathrm{~kb}$ )

\section{Abbreviations}

$\mathrm{BHI}$ : Brain, heart, infusion agar; Credence RID ${ }^{\mathrm{TM}}$ : Credence Rapid Infection Detection $^{\text {TM}}$; HMP: Human Microbioime Project; NBG: No bacterial growth; NCBI: National Centre for Biotechnology Information; NGS: Next generation sequencing; OT2: One Touch Two ${ }^{\mathrm{TM}}$; PCR: Polymerase chain reaction; PGM: Personal Genome Machine ${ }^{\text {TM}}$; PPCN: PCR positive, culture negative; SIDCER: Strategic Initiative for Developing Capacity in Ethical Review

\section{Acknowledgments}

Nawaloka Metropolis Laboratory for provision of the clinical material for the study and the culture results for the final comparison.

\section{Funding}

This study is industry sponsored, with funding provided by Credence Genomics Pvt. Ltd.

\section{Availability of data and materials}

The data sets supporting the conclusions of this article are included within the article and its additional files.

\section{Authors' contributions}

VSG provided clinical input and overall guidance on the development of the study design and final analysis of results. JP provided assistance in coordinating the samples collection, clinical interpretation of the output of sequencing results and contributed to the writing of the manuscript. VC provided expertise on molecular biology, input on batch handling for NGS analysis and advise on molecular based interpretation of results. LMA, NAU, DSL, BNE, JVSA, CST, DPT, TK, IJ and SA were involved in the overall NGS analysis process from sample collection, DNA extraction, library preparation, template preparation, sequencing, bioinformatics, data analysis and results comparison. LMA was a major contributor to the final manuscript. All authors have read and approved the final manuscript.

\section{Ethics approval and consent to participate}

Though human derived samples will be used, no human DNA from the patients will be used in this study. Specimens will not be expressly collected for this study but rather remnants of commercial culture specimens will be used for validation. Only bacterial or fungal DNA isolated from these specimens will be used in the study.

Application for ethical review was submitted to SICER accredited Ethical Review Committee, Faculty of Medicine, University of Colombo (Reference EC-16-134). Study protocol was approved on 11th August 2016.

\section{Consent for publication}

Not Applicable.

\section{Competing interests}

Financial: This study was sponsored by Credence Genomics Pvt. Ltd. for validation of commercial NGS-based testing product. Author VSG is the CEO/ Managing Director of Credence Genomics Pvt. Ltd.

Authors LMA, NAU, BNE, CST are employees of Credence Genomics Pvt. Ltd. SA, DSL, JVSA, DPT, TK and IJ are past employees of Credence Genomics Pvt. Ltd.

Author VC is affiliated with Credence Genomics Pvt. Ltd. as a consultant in molecular biology.

Author J.P is affiliated with the University of Colombo as the Professor of Microbiology, and specialist Medical Microbiologist at Nawaloka Metropolis laboratory. JP is also affiliated with Credence Genomics Pvt. Ltd. as a specialist in Medical Microbiology.

\section{Publisher's Note}

Springer Nature remains neutral with regard to jurisdictional claims in published maps and institutional affiliations. 


\section{Author details}

'Dean and Chair Professor of Microbiology, Faculty of Medicine, University of Colombo, P.O. box 271, Colombo, Sri Lanka. 'Department of Chemistry, Faculty of Science, University of Colombo, Colombo, Sri Lanka. ${ }^{3}$ Credence Genomics Pvt. Ltd, 12 - 3/2, Sunethradevi Road, Kohuwala, Nugegoda, Sri Lanka.

Received: 30 May 2017 Accepted: 12 September 2017

\section{Published online: 19 September 2017}

\section{References}

1. The Human Microbiome. Human Microbiome RSS. Available from: https:// www.hmpdacc.org/overview/about/. Accessed on 5 Feb 2017.

2. World Health Organization. The top 10 causes of death. Available from: http:// www.who.int/mediacentre/factsheets/fs310/en/. Accessed on 16 Feb 2017.

3. Stewart EJ. Growing Unculturable bacteria. J Bacteriol. 2012;194:4151-60.

4. Nishie M, Nagao J-I, Sonomoto K. Antibacterial peptides "Bacteriocins": an overview of their diverse characteristics and applications. Biocont Sci. 2012; 17(1):1-16.

5. Lane DJ, Pace B, Olsen GJ, Stahl DA, Sogin ML, Pace NR. Rapid determination of 165 ribosomal RNA sequences for phylogenetic analyses. Proc Nat Acad Sci. 1985;82(20):6955-9.

6. Clarridge JE. Impact of $16 \mathrm{~S}$ rRNA gene sequence analysis for identification of bacteria on clinical microbiology and infectious diseases. Clin Microbiol Rev. 2004;17(4):840-62.

7. Petti CA, Polage CR, Schreckenberger $P$. The role of 165 rRNA gene sequencing in identification of microorganisms misidentified by conventional methods. J Clin Microbiol. 2005;43(12):6123-5.

8. Leaw SN, Chang HC, Sun HF, Barton R, Bouchara J-P, Chang TC. Identification of medically important yeast species by sequence analysis of the internal transcribed spacer regions. J Clin Microbiol. 2006;44(3):693-9.

9. Thomas T, Gilbert J, Meyer F. Metagenomics - a guide from sampling to data analysis. Micro Infor Experi. 2012;2(1):3.

10. Handelsman J. Metagenomics: application of genomics to uncultured microorganisms. Microbiol Mol Biol Rev. 2004;68(4):669-85.

11. Salipante SJ, Sengupta DJ, Rosenthal C, Costa G, Spangler J, Sims EH, et al. Rapid $16 \mathrm{~S}$ rRNA next-generation sequencing of Polymicrobial clinical samples for diagnosis of complex bacterial infections. PLoS One. 2013;8(5):e65226.

12. Korabecna M. The Variability in the Fungal Ribosomal DNA (ITS1, ITS2, and 5.8 S rRNA Gene): Its Biological Meaning and Application in Medical Mycology. In: Communicating Current Research and Educational Topics and Trends in Applied Microbiology. $1^{\text {st }}$ Edition. Formatex (Spain); 2007. p. 7837.

13. Cowan ST, Steel KJ, Barrow Gl, Feltham RKA. Cowan and Steel's manual for the identification of medical bacteria. 3rd ed. Cambridge: Cambridge University Press; 1993.

14. Jorgensen J, Pfaller M, Carroll K, Funke G, Landry M, Richter S, et al. Manual of clinical microbiology. 11th ed. Washington, DC: ASM Press; 2015.

15. Federicks DN, Relman DA. Improved amplification of microbial DNA from blood cultures by removal of the PCR inhibitor sodium Polyanetholesulfonate. J Clin Microbiol. 1998;36(10):2810-6.

16. Fournier P-E, Raoult D. Prospects for the future using genomics and proteomics in clinical microbiology. Ann Rev Microbiol. 2011;65(1):169-88.

17. Bello CSS, Qahtani A. Pitfalls in the routine diagnosis of Staphylococcus Aureus. Afri J Biotech. 2005;4(1):83-6.

18. Hardy DR, Octavio R. Mycoplasma infections. In: Infectious diseases of the fetus and newborn infant. 6th ed. Philadelphia: W.B. Saunders; 2006. p. 499-512.

19. Cunningham SA, Mandrekar JN, Rosenblatt JE, Patel R. Rapid PCR detection of Mycoplasma hominis, Ureaplasma urealyticum, and Ureaplasma parvum. Int J Bacteriol. 2013;2013:1-7.

20. Decuypere S, Meehan CJ, Puyvelde SV, Block TD, Maltha J, Palpouguini L, et al. Diagnosis of bacterial bloodstream infections: a 165 Metagenomics approach. PLoS Negl Trop Dis. 2016;10(2):e0004470.

21. Sarookhani MR, Ayazi P, Alizadeh S, Foroughi F, Sahmani A, Adineh M. Comparison of $16 \mathrm{~S}$ rDNA-PCR amplification and culture of cerebrospinal fluid for diagnosis of bacterial meningitis. Iran J Ped. 2010;20(4):471-5.
22. Rampini SK, Bloemberg GV, Keller PM, Buchler AC, Dollenmaier G, Speck RF, et al. Broad-range 16S rRNA gene polymerase chain reaction for diagnosis of culture-negative bacterial infections. Clin Infec Dis. 2011;53(12):1245-51.

23. NCBI Reference Sequence (RefSeq) Database. Available from: ftp://ftp.ncbi. nlm.nih.gov/refseq/release/release-notes/archive/RefSeq-release80.txt. Accessed 10 Jan 2017.

\section{Submit your next manuscript to BioMed Central and we will help you at every step:}

- We accept pre-submission inquiries

- Our selector tool helps you to find the most relevant journal

- We provide round the clock customer support

- Convenient online submission

- Thorough peer review

- Inclusion in PubMed and all major indexing services

- Maximum visibility for your research

Submit your manuscript at www.biomedcentral.com/submit
Biomed Central 